\title{
INCIDENCE DIMENSION AND 2-PACKING NUMBER IN GRAPHS
}

\author{
Dragana Božović ${ }^{1,2}$, Aleksander Kelenc ${ }^{1,2}$, Iztok Peterin Ir, $^{1,2}$ \\ AND ISMAEL G. YERO ${ }^{3}$
}

\begin{abstract}
Let $G=(V, E)$ be a graph. A set of vertices $A$ is an incidence generator for $G$ if for any two distinct edges $e, f \in E(G)$ there exists a vertex from $A$ which is an endpoint of either $e$ or $f$. The smallest cardinality of an incidence generator for $G$ is called the incidence dimension and is denoted by $\operatorname{dim}_{I}(G)$. A set of vertices $P \subseteq V(G)$ is a 2-packing of $G$ if the distance in $G$ between any pair of distinct vertices from $P$ is larger than two. The largest cardinality of a 2-packing of $G$ is the packing number of $G$ and is denoted by $\rho(G)$. In this article, the incidence dimension is introduced and studied. The given results show a close relationship between $\operatorname{dim}_{I}(G)$ and $\rho(G)$. We first note that the complement of any 2-packing in graph $G$ is an incidence generator for $G$, and further show that either $\operatorname{dim}_{I}(G)=|V(G)-| \rho(G)$ or $\operatorname{dim}_{I}(G)=|V(G)-| \rho(G)-1$ for any graph $G$. In addition, we present some bounds for $\operatorname{dim}_{I}(G)$ and prove that the problem of determining the incidence dimension of a graph is NP-hard.
\end{abstract}

Mathematics Subject Classification. 05C69, 05C12.

Received May 15, 2019. Accepted January 3, 2022.

\section{INTRODUCTION}

The famous Gallai's theorem states that

$$
\alpha(G)+\beta(G)=n,
$$

where $G$ is a graph on $n$ vertices, $\beta(G)$ is the vertex covering number of $G$ and $\alpha(G)$ is the independence number of $G$. Its beauty does not only lie in the numbers. Any vertex cover set $S$ of cardinality $\beta(G)$ determines an independent set $I$ of cardinality $\alpha(G)$, which is the complement of $S$. With such elegance, the hunt for analog results is always open.

One possibility arises from observing an independent set from a different (but equivalent) perspective. It comes from the notion of $k$-packings in graphs. A set $P \subseteq V(G)$ is a $k$-packing set (or $k$-packing for short) of $G$ if the distance between any pair of distinct vertices from $P$ is greater than $k$. The $k$-packing number of $G$ is the maximum cardinality of any $k$-packing of $G$ and is denoted by $\rho_{k}(G)$. Clearly, 1-packings represent independent

Keywords. incidence dimension, incidence generator, 2-packing.

1 Faculty of Electrical Engineering and Computer Science, University of Maribor, Koroška cesta 46, 2000 Maribor, Slovenia.

2 Institute of Mathematics, Physics and Mechanics, Jadranska ulica 19, 1000 Ljubljana, Slovenia.

3 Departamento de Matemáticas, Escuela Técnica Superior de Ingeniería de Algeciras, Universidad de Cádiz, Av. Ramón Puyol s/n, 11202 Algeciras, Spain.

*Corresponding author: iztok.peterin@um.si

(C) The authors. Published by EDP Sciences, ROADEF, SMAI 2022 
sets of $G$, and maximum 1-packings are maximum independent sets of $G$. A $\rho_{k}(G)$-set is a packing of cardinality $\rho_{k}(G)$. Since we are interested only in 2-packings, from now on, we will use the terminology $\rho(G)$, instead of $\rho_{2}(G)$, and packing, instead of 2-packing. A natural question concerns finding an analogy to Gallai's theorem for maximum $k$-packings for $k \geq 2$. We deal with this problem for $k=2$.

For a longer period of time packings were considered as a natural lower bound for the domination number of graphs (for definitions, terminology and more information on domination in graphs we suggest the books $[12,13])$. One of the first results (and indeed very remarkable) of that type is from Meir and Moon [24], who have shown that $\rho(T)=\gamma(T)$ for every tree $T$ (using different notation). Efficient closed domination graphs represent a class of graphs with $\rho(G)=\gamma(G)$, where both maximum 2-packing sets and minimum dominating sets coincide. In such a case, we call a minimum dominating set a 1-perfect code. The study of perfect codes in graphs was initiated by Biggs [1]. Later it was intensively studied. We recommend [19] for further information and references.

In the last decade, the packing number became more interesting, not only in connection with the domination number. For instance, the relationship between the packing number and maximal packings of minimum cardinality, called also the lower packing number, is investigated in [27]. A connection (in the form of an upper bound) between the packing number and the double domination is presented in [25]. Graphs for which their packing number equals the packing number of their complement are described in [4]. Henning et al. [14] shows that the domination number is bounded from above by the packing number multiplied with the maximum degree of a graph.

A generalization of packing, called $k$-limited packing, is presented in [8]. Here every vertex can have at most $k$ neighbors in a $k$-limited packing set $S$. To achieve some bounds, a probabilistic approach to $k$-limited packings was introduced in [7]. A further generalization of it is shown in [3]. It brings a dynamic approach concerning the vertices of $G$, where different vertices can have a different number of neighbors in a generalized limited packing. As shown in [3], the problem of computing the packing number of graphs is NP-hard but polynomially solvable for $P_{4}$-tidy graphs.

Our goal is to continue finding several contributions on packings in connection with other topics in graphs. In recent years, this has attracted several researchers' attention who also tried to find the connections with the parameters close to metric dimension. The metric dimension of graph $G$ (introduced first in [11,28]) and its large number of variants are due to their properties of uniquely recognizing (identifying or determining) vertices or edges of $G$ nowadays largely studied. Some of the most recent variants deal with uniquely identifying the edges of a considered graph (which is in some way one of the ideas that motivated this contribution). The first works on these recent topics are $[17,18]$. Another metric parameter that can be taken as a predecessor of the present study concerns identification of vertices throughout neighborhoods (see [15]). We next describe all of these related concepts.

Given graph $G$, a set $S$ of vertices of $G$ is an adjacency generator ${ }^{1}$ for $G$ if for any two different vertices $u, v \in V(G)-S$, there exists $x \in S$ such that $|N(x) \cap\{u, v\}|=1$. An adjacency generator of minimum cardinality is called an adjacency basis for $G$. Its cardinality is the adjacency dimension of $G$ and is denoted by $\operatorname{dim}_{A}(G)$. These concepts were first introduced in [15] as a tool while studying some metric properties of the lexicographic product of graphs. More results on the adjacency dimension of graphs can be found in $[5,6]$.

Given a vertex $v \in V(G)$ and an edge $e=u w \in E(G)$, the distance between the vertex $v$ and the edge $e$ is defined as $d(e, v)=\min \{d(u, v), d(w, v)\}$. A vertex $w \in V(G)$ distinguishes two edges $e_{1}, e_{2} \in E(G)$ if $d\left(w, e_{1}\right) \neq d\left(w, e_{2}\right)$. A nonempty set $S \subset V(G)$ is an edge metric generator for $G$ if any two edges of $G$ are distinguished by some vertex of $S$. An edge metric generator with the smallest possible cardinality is called an edge metric basis for $G$. Its cardinality is the edge metric dimension and is denoted by $\operatorname{edim}(G)$. This concept was introduced in [18]. Some other studies on the edge metric dimension of graphs appeared in [10,20-22,26,30]. Moreover, for some extra information on these parameters, and several other works on the metric dimension of

\footnotetext{
${ }^{1}$ In fact, these sets were called adjacency resolving sets in [15], where the concept was first described.
} 
graphs and its variants, in particular for the ones identifying edges of graphs, we suggest the two recent surveys $[23,29]$.

As a kind of a mix with respect to the last two parameters, we introduce the concept of incidence dimension in graphs which arises from the two concepts above in some natural way of research evolution. We formally define it in the next section. The definition is based on the existence of some properties of the complement of a packing set. Then we present a connection between the incidence dimension and packing number of graphs. A section about the complexity of the incidence dimension follows. We conclude this work with some additional information about the incidence dimension.

We consider only finite, undirected and simple graphs. Let $G$ be a graph with vertex set $V(G)$ and edge set $E(G)$. For a fixed $v \in V(G)$, set $\{u \in V(G): u v \in E(G)\}$ represents the open neighborhood of $v$ and is denoted by $N(v)$. The degree of $v$ is $d(v)=|N(v)|$. The closed neighborhood of $v \in V(G)$ is $N[v]=N(v) \cup\{v\}$. The distance $d(u, v)$ between any two vertices $u$ and $v$ is the minimum number of edges on a path between them. Given a set of vertices $S$ of $G$, we use $G-S$ to denote the graph obtained from $G$ by removing all the vertices of $S$ and the edges incident with them. If $S=\{v\}$ for some vertex $v$, then we simply write $G-v$. The subgraph of $G$ induced by $D \subset V(G)$ will be denoted by $G[D]$.

\section{DEFINING THE INCIDENCE DIMENSION AND ITS CONNECTION WITH PACKING NUMBER}

As mentioned in the introduction, we are interested in some properties of the complement of the packing sets of graph $G$. The following result provides a motivation for the definition of the incidence dimension.

Proposition 2.1. If a set $X \subseteq V(G)$ is a packing set of graph $G$, then the set $S=V(G) \backslash X$ is a vertex cover of $G$ and for any two different edges $e$ and $f$ there exists $x \in S$ such that either $x$ is incident with $e$ or $x$ is incident with $f$.

Proof. First, let $e=u v$ be an arbitrary edge of $G$. If $\{u, v\} \cap S=\emptyset$, then $u, v \in X$. Since $d(u, v)=1$, this yields a contradiction with $X$ being a packing set of $G$. Thus, $S$ is a vertex cover. (This also follows from the fact that every packing is also a 1-packing and, with this, an independent set. Since the complements of independent sets are vertex covers, the result follows.)

Take now two different arbitrary edges $e, f \in E(G)$. If $e=u v$ and $f=a b$ are not incident, then they are distinguished by one endpoint of $e$ which exists in $S$ because $S$ is a vertex cover. Otherwise they are incident in one vertex, say in $u=a$. If $\{v, b\} \cap S=\emptyset$, then $v, b \in X$. This is a contradiction with $X$ being a packing of $G$ as $d(b, v) \leq 2$. So at least one of $v$ or $b$, say $v$, is in $S$ and $v$ is the desired vertex.

With the second property of the above proposition, we are able to define the incidence dimension as follows. For this, note that it is meaningful to demand a minimum cardinality set with this mentioned property in order to retain the analogy with the relationship between independence number and vertex cover number.

Definition 2.2. Given two edges $e, f \in E(G)$ and a vertex $x \in V(G)$, we say that $x$ (incidently) resolves or distinguishes the pair $e, f$ if either $x \in e$ or $x \in f$ (exactly one of these two edges is incident with $x$ ). A set $S$ of vertices of $G$ is an incidence generator for $G$ if for any two different edges $e, f \in E(G)$ there exists a vertex $x \in S$ such that $x$ incidently resolves the pair $e, f$. An incidence generator of minimum cardinality is called an incidence basis for $G$, and its cardinality, denoted by $\operatorname{dim}_{I}(G)$, is the incidence dimension of $G$.

From this, we can immediately see that we cannot expect such an elegant result as in the case of Gallai's theorem. Indeed, we can see that for $K_{2}$, an empty set is an incidence generator of minimum cardinality since there exists only one edge in $K_{2}$, and we have $\operatorname{dim}_{I}\left(K_{2}\right)=0$. Clearly, this can be extended to any graph with only one edge. However, as soon as there are two edges in $G$, we have $\operatorname{dim}_{I}\left(K_{2}\right)>0$.

The next observation is that if $S$ is an incidence generator for $G$, then there exists at most one edge with both endpoints outside of $S$. Namely, two such edges would not be incidently resolved by $S$, a contradiction with $S$ being an incidence generator for $G$. 


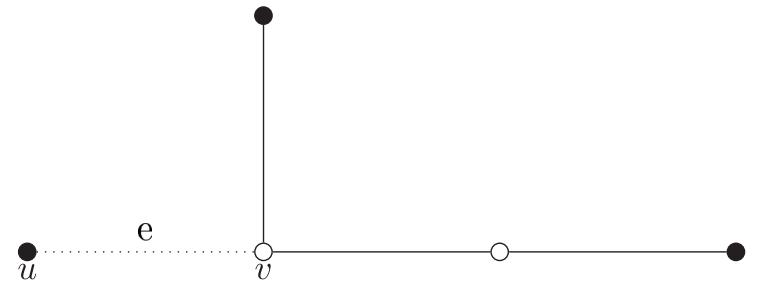

Figure 1. Graph $G$ and edge $e$ for which it holds that $\rho(G-e)>\left|P_{e}(G)\right|$.

Before we state a deeper connection between the incidence dimension and the packing number of a graph, we need some additional terminology.

Definition 2.3. Let $e=u v$ be an edge of graph $G$. The $e$-critical packing of $G-e$, denoted by $P_{e}(G)$, is a maximum packing of the graph $G-e$ with the following property.

$$
\text { If }\left|\{u, v\} \cap P_{e}(G)\right|<2 \text {, then } P_{e}(G) \text { is a packing of } G \text {. }
$$

Notice that both $u$ and $v$ can be in $P_{e}(G)$, and (2.1) is trivially fulfilled. Clearly, $P_{e}(G)$ is not a packing of $G$ in such a case. However, by removing either $u$ or $v$ from $P_{e}(G)$, we obtain a packing of $G$. If $u, v \notin P_{e}(G)$, the set $P_{e}(G)$ is also a packing for $G$. If exactly one endpoint of $e$, say $u$, is inside $P_{e}(G)$, then $N_{G}(v) \cap P_{e}(G)=\{u\}$ or else $P_{e}(G)$ is not a packing of $G$ contradicting (2.1). Therefore we have

$$
\rho(G) \leq\left|P_{e}(G)\right| \leq \rho(G)+1 .
$$

Figure 1 shows an example of graph $G$, which for the drawn dashed edge has an $e$-critical packing smaller than $\rho(G-e)$. Black vertices represent the unique maximum packing of $G-e$, which does not fulfill (2.1) and is therefore not $e$-critical. Hence, the cardinality of every $e$-critical packing is two.

Theorem 2.4. If $G$ is a graph of order $n$ and $k$ is an integer defined as $k=\max _{e \in E(G)}\left|P_{e}(G)\right|$, then $\operatorname{dim}_{I}(G)=$ $n-k$. An incidence basis for the graph $G$ is any set $S=V(G) \backslash P_{e}(G)$ for which it holds that $k=\left|P_{e}(G)\right|$.

Proof. Let $e=u v$ and $P=P_{e}(G)$ such that $k=\left|P_{e}(G)\right|$. We want to prove that $S=V(G) \backslash P$ is an incidence generator for $G$.

If $|\{u, v\} \cap P|<2$, then $P$ is also a packing of $G$ by (2.1). Due to Proposition 2.1, $S$ is an incidence generator for $G$. Otherwise $u, v \in P$. Hence, $P$ is a packing of the graph $G-e$. Due to Proposition 2.1, $S$ is an incidence generator for $G-e$ with the property that every edge of $G-e$ has at least one endpoint in $S$. Since $S$ is an incidence generator for $G-e$, we have to consider only the pairs of edges where one edge is $e$. Take edge $e$ and an arbitrary edge $f \neq e$ of $G$. Clearly, the edges $e$ and $f$ are distinguished by the endpoint of $f$ that is in $S$. It follows that $S$ is an incidence generator for $G$ and $\operatorname{dim}_{I}(G) \leq n-k$.

Now suppose that there exists an incidence generator $S^{\prime}$ for $G$ with cardinality $\left|S^{\prime}\right|=d^{\prime}<n-k$. Since $S^{\prime}$ is an incidence generator for $G$, it follows that there exists at most one edge in $G$ induced by $P_{e}^{\prime}(G)=V(G) \backslash S^{\prime}$. Suppose that such an edge $e=u v$ exists. First, notice that in $G-e$ there is no edge between two vertices of $P_{e}^{\prime}(G)$. Since $S^{\prime}$ is an incidence generator for $G$, there do not exist two arbitrary vertices $x, y \in P_{e}^{\prime}(G)$ such that $d_{G-e}(x, y)=2$. Thus, it follows that $P_{e}^{\prime}(G)$ is a packing for $G-e$ and both $u, v \in P_{e}^{\prime}(G)$. Since the cardinality of $P_{e}^{\prime}(G)$ is $n-d^{\prime}>k$, we obtain a contradiction with the maximality of $P_{e}(G)$. If there is no edge in the graph induced by $P_{e}^{\prime}(G)$, then $P_{e}^{\prime}(G)$ is a packing of $G$ by (2.1). Every packing of $G$ is also a packing of $G-e$ with property (2.1) for an arbitrary edge $e$. Again, this is a contradiction with the maximality of $P_{e}(G)$. Therefore, we deduce that there is no incidence generator with cardinality less than $n-k$. 
A direct consequence of Theorem 2.4, together with (2.2), follows.

Corollary 2.5. For every graph $G$ of order $n$ it holds that $n-\rho(G)-1 \leq \operatorname{dim}_{I}(G) \leq n-\rho(G)$.

This yields a natural partition of graphs into two classes, those whose incidence dimension equals $|V(G)|-$ $\rho(G)-1$ and those for which $\operatorname{dim}_{I}(G)=|V(G)|-\rho(G)$. To show that graph $G$ belongs to the first class, we need to find an edge $e$ such that $\left|P_{e}(G)\right|=\rho(G)+1$. For the second class, we need to show that for each edge $e$, we have $\left|P_{e}(G)\right|=\rho(G)$.

We next derive exact results for the incidence dimension for some graph families, and we first consider a class of graphs called edge-triangular. A graph is called edge-triangular if every edge of a graph is in at least one 3-cycle.

Proposition 2.6. Let $S$ be any incidence generator for graph $G$. Then the graph $G$ is edge-triangular if and only if for every $e=u v \in E(G)$ it holds that $|\{u, v\} \cap S|>0$.

Proof. Let $G$ be an edge-triangular graph. Suppose there exists en edge $e=u v$ and an incidence generator $S$ for $G$ such that $|\{u, v\} \cap S|=0$. Since $G$ is an edge-triangular graph, there exists a vertex $w$ such that $u v w u$ is a triangle. Note that the vertex $w$ has to be in $S$ because $e$ and $u w$ have to be distinguished by at least one endpoint. However, the edges $u w$ and $v w$ are not distinguished by any vertex from $S$. This is a contradiction with $S$ being an incidence generator.

Conversely, suppose that the graph $G$ is not edge-triangular. Thus, there exists an edge $e=u v$ that is not a part of a triangle. Consequently, the set $S=V(G) \backslash\{u, v\}$ is an incidence generator for $G$, which means $|\{u, v\} \cap S|=0$.

Proposition 2.6 implies the following corollary.

Corollary 2.7. Let $G$ be an edge-triangular graph. The set $S$ is an incidence generator for $G$ if and only if $V(G) \backslash S$ is a packing of $G$.

Proof. Suppose that $S$ is an incidence generator. Due to Proposition 2.6 there are no two vertices at distance 1 in $V(G) \backslash S$. Since $S$ is an incidence generator, there are also not two vertices at distance 2 in $V \backslash S$. It follows that $V(G) \backslash S$ is a packing of $G$.

By Proposition 2.1, the converse holds for any graph $G$.

Proposition 2.8. Let $n, r$ and $t$ be integers.

(i) If $n \geq 3$, then $\operatorname{dim}_{I}\left(K_{n}\right)=n-1$.

(ii) If $n \geq 3$, then $\operatorname{dim}_{I}\left(P_{n}\right)=\left\lfloor\frac{2(n-1)}{3}\right\rfloor$.

(iii) If $n \geq 4$, then $\operatorname{dim}_{I}\left(C_{n}\right)=\left\lfloor\frac{2 n}{3}\right\rfloor$.

(iv) If $r, t \geq 1$, then $\operatorname{dim}_{I}\left(K_{r, t}\right)=r+t-2$.

Proof. (i) Clearly, $K_{n}$ is an edge-triangular graph. So, the result follows from Corollary 2.7, and the fact that any set containing all but one vertex of any graph $G$ is an incidence generator for $G$. Such an incident generator is minimum because $V\left(K_{n}\right)-\{x, y\}$ is not an incident generator for different $x, y \in V\left(K_{n}\right)$ as edges $x w$ and $y w$ are not distinguished by $V\left(K_{n}\right)-\{x, y\}$ for any $w \in V\left(K_{n}\right)-\{x, y\}$.

(ii) Let $V\left(P_{n}\right)=\left\{v_{0}, v_{1}, \ldots, v_{n-1}\right\}$ such that $v_{i} v_{i+1} \in E\left(P_{n}\right)$ for every $i \in\{0, \ldots, n-2\}$. Consider the set $S^{\prime}=\left\{v_{i}: i \geq 2\right.$ and $i \equiv 0$ or $\left.i \equiv 2(\bmod 3)\right\}$. Note that any two edges of $P_{n}$ are incidently resolved by $S^{\prime}$, and so $\operatorname{dim}_{I}\left(P_{n}\right) \leq\left|S^{\prime}\right|=\left\lfloor\frac{2(n-1)}{3}\right\rfloor$.

On the other hand, let $S$ be an incidence basis for $P_{n}$. There could be at most one edge which is not incident to any vertex of $S$. Also, if $v_{i} \in S$ and $i \neq 0, n-1$, then $v_{i-1} \in S$ or $v_{i+1} \in S$ or both. Thus, at least two of any 


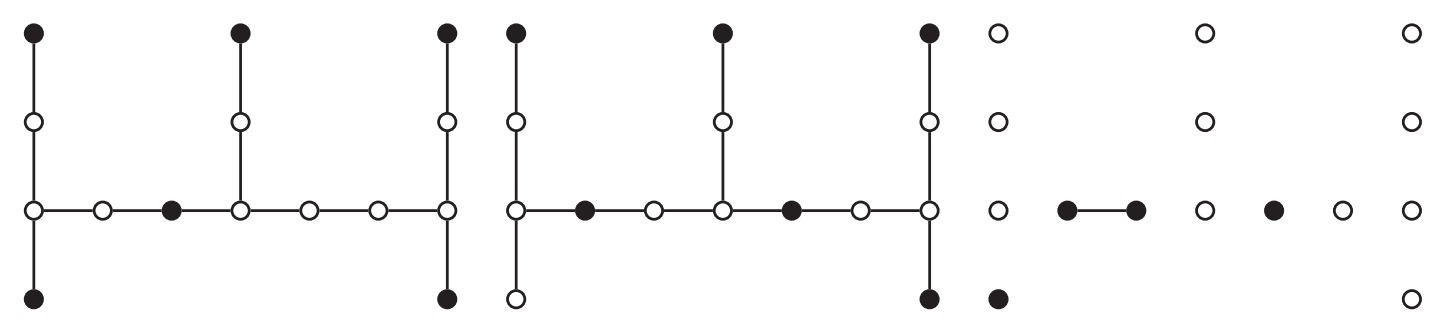

FiguRE 2. An example showing that the converse implication of Theorem 2.9 does not hold in general.

three consecutive vertices $v_{i}, v_{i+1}, v_{i+2}$ are in $S$. According to these facts, $\operatorname{dim}_{I}\left(P_{n}\right)=|S| \geq\left\lfloor\frac{2(n-1)}{3}\right\rfloor$, which completes the proof of (ii).

(iii) Let $e=u v$ be any edge of $C_{n}$. Clearly, $C_{n}-e \cong P_{n}$. It is well known, see [24], that $\rho(T)=\gamma(T)$ for every tree $T$ and we have $\rho\left(P_{n}\right)=\left\lceil\frac{n}{3}\right\rceil$. Moreover, there always exists a $\rho\left(P_{n}\right)$-set $P$ such that $u, v \in P$. Hence, $\left|P_{e}\left(C_{n}\right)\right|=\left\lceil\frac{n}{3}\right\rceil$. On the other hand, $\rho\left(C_{n}\right)=\left\lfloor\frac{n}{3}\right\rfloor$. If $n \neq 3 k$, then $\left|P_{e}\left(C_{n}\right)\right|=\rho\left(C_{n}\right)+1$, and by Theorem 2.4, we have $\operatorname{dim}_{I}\left(C_{n}\right)=n-\left\lceil\frac{n}{3}\right\rceil=\left\lfloor\frac{2 n}{3}\right\rfloor$. For $n=3 k$ we have $\left|P_{e}\left(C_{n}\right)\right|=\rho\left(C_{n}\right)=k$ for every edge $e$ and, again by Theorem 2.4, we have $\operatorname{dim}_{I}\left(C_{n}\right)=n-\rho\left(C_{n}\right)=3 k-k=\frac{2 n}{3}=\left\lfloor\frac{2 n}{3}\right\rfloor$. So, we are done with the proof of (iii).

(iv) Clearly $P_{e}\left(K_{r, t}\right)=\{u, v\}$ for any edge $e=u v$ of $K_{r, t}$, while $\rho\left(K_{r, t}\right)=1$. By Corollary 2.5 and a comment after it we have $\operatorname{dim}_{I}\left(K_{r, t}\right)=r+t-2$, because all edges are symmetric to each other.

Recall that $A \triangle B$ denotes the symmetric difference of the sets $A$ and $B$.

Theorem 2.9. Let $G$ be a graph. If $\operatorname{dim}_{I}(G)=|V(G)|-\rho(G)-1$, then $G\left[P_{1} \triangle P_{2}\right]$ is not an empty graph for some maximum packings $P_{1}$ and $P_{2}$ of $G$.

Proof. Let $G$ be a graph and let $S$ be an incidence basis of $G$ such that $\operatorname{dim}_{I}(G)=|V(G)|-\rho(G)-1$. Let $P=V(G)-S$. Since $|P|=\rho(G)+1, P$ is not a packing for $G$. So, there exist two different vertices $u, v \in P$, such that $1 \leq \mathrm{d}(u, v) \leq 2$. If $\mathrm{d}(u, v)=2$, then the edges $u w$ and $w v$, where $w$ is a common neighbour of $u$ and $v$, are not resolved by $S$, a contradiction. Thus d $(u, v)=1$. Let $P_{1}=V(G)-(S \cup\{u\})$ and $P_{2}=V(G)-(S \cup\{v\})$. The cardinality of both packings is maximum possible, since $\left|P_{1}\right|=\left|P_{2}\right|=|V(G)|-((|V(G)|-\rho(G)-1)+1)=$ $\rho(G)$. Since $u$ and $v$ are adjacent it follows that $G\left[P_{1} \triangle P_{2}\right]$ is not an empty graph and the proof is completed.

The converse implication of Theorem 2.9 does not hold in general, as we can see from the example in Figure 2. Two different packings of $G$ are presented by black vertices on the left and in the middle of Figure 2. On the right side, there is a picture of $G\left[P_{1} \triangle P_{2}\right]$, which is clearly not an empty graph. However, the incidence dimension of $G$ is not equal to $|V(G)|-\rho(G)-1$ because one can check that $P_{e}(G)=\rho(G)$ for every edge $e \in E(G)$ as mentioned in a comment after Corollary 2.5.

We next provide an exact result for the class of graphs with the unique maximum packing. Two characterizations of trees with the unique maximum packing were presented recently in [2].

Theorem 2.10. If $G$ is a graph with the unique maximum packing $P$, then $\operatorname{dim}_{I}(G)=|V(G)|-\rho(G)$.

Proof. Let $G$ be a graph and $P$ its unique maximum packing. To prove that $\operatorname{dim}_{I}(G)=|V(G)|-\rho(G)$, we will use the contraposition of Theorem 2.9: If $G\left[P_{1} \triangle P_{2}\right]$ is an empty graph, then $\operatorname{dim}_{I}(G) \neq|V(G)|-\rho(G)-1$. It follows that $P_{1}=P_{2}=P$, because $P$ is a unique maximum packing of $T$. So, $G\left[P_{1} \triangle P_{2}\right]$ is an empty graph for any maximum packings $P_{1}$ and $P_{2}$ and $\operatorname{dim}_{I}(G) \neq|V(G)|-\rho(G)-1$. By Corollary 2.5 we conclude that $\operatorname{dim}_{I}(G)=|V(G)|-\rho(G)$. 


\section{Complexity of the Problem}

In this section, we consider the computational complexity of computing the incidence dimension of a graph. We show that the problem of finding the incidence dimension of an arbitrary graph is NP-hard. For this, we strongly rely on edge-triangular graphs. We consider the following decision problem.

$$
\begin{aligned}
& \text { INCIDENCE DIMENSION PROBLEM (IDIM problem for short) } \\
& \text { INSTANCE: Graph } G \text { of order } n \geq 3 \text { and an integer } 1 \leq r \leq n-1 \text {. } \\
& \text { QUESTION: } \operatorname{Is}_{I}(G) \leq r \text { ? }
\end{aligned}
$$

To study the complexity of IDIM problem, we make a reduction from 3-SAT problem, which is one of the most classical NP-complete problems known in the literature. For more information on 3-SAT problem and reducibility of NP-complete problems in general, we suggest [9].

Theorem 3.1. IDIM problem is NP-complete.

Proof. For a set of vertices $S$ guessed for the problem by a nondeterministic algorithm, one needs to iterate through all pairs of edges and check that every pair is incidently resolved by a vertex from $S$. This can be done in polynomial time, and therefore IDIM problem is in NP. We make a polynomial transformation of 3-SAT problem to IDIM problem in the following way. Consider an arbitrary instance of 3-SAT problem, i.e., a finite set $U=\left\{u_{1}, \ldots, u_{n}\right\}$ of Boolean variables and a collection $C=\left\{c_{1}, \ldots, c_{m}\right\}$ of clauses, each containing three Boolean variables from $U$. We will construct graph $G=(V, E)$ and set a positive integer $r \leq|V|-1$, such that $\operatorname{dim}_{I}(G) \leq r$ if and only if $C$ is satisfiable. The construction will be made up of several gadgets and edges between them.

For each variable $u_{i} \in U, 1 \leq i \leq n$, we construct a truth-setting gadget $X_{i}=\left(V_{i}, E_{i}\right)$, with $V_{i}=$ $\left\{x_{i}, y_{i}, z_{i}, w_{i}, T_{i}, F_{i}\right\}$ and $E_{i}=\left\{x_{i} y_{i}, x_{i} z_{i}, y_{i} z_{i}, y_{i} w_{i}, z_{i} w_{i}, w_{i} T_{i}, w_{i} F_{i}, T_{i} F_{i}\right\}$, see Figure 3. Each truth-setting gadget is connected to the rest of the graph only through nodes $T_{i}$ and $F_{i}$, which are representing values TRUE and FALSE, respectively.

Claim. Let $u_{i}$ be an arbitrary variable in $U$. Any incidence generator $S$ must contain at least four vertices from its truth-setting gadget. Moreover, if there are exactly four vertices from a truth-setting gadget in $S$, then $y_{i}, w_{i}, z_{i} \in S$ and $x_{i} \notin S$.

Proof. Towards contradiction suppose that there exists an incidence generator $S$ with less than four vertices from the truth-setting gadget corresponding to $u_{i}$. It follows that there exists a set of three vertices $W_{i}=$ $\left\{v_{1}, v_{2}, v_{3}\right\} \subset V_{i}$ that are not in $S$. Make a partition of $V_{i}$ into two sets $V_{i}=\left\{x_{i}, y_{i}, z_{i}\right\} \cup\left\{w_{i}, T_{i}, F_{i}\right\}$. There are at least two vertices from $W_{i}$ in one of the partition sets. Since each partition set forms a triangle, it follows that there is an edge lying outside $S$, a contradiction with Proposition 2.6.

Suppose now that exactly four vertices from a truth-setting gadget are in $S$. If $w_{i} \notin S$, then $T_{i}, F_{i}, y_{i}, z_{i} \in S$ because otherwise we have a contradiction with Proposition 2.6. But then $x_{i} \notin S$ and edges $w_{i} z_{i}$ and $x_{i} z_{i}$ are not distinguished by $S$, a contradiction. Hence, $w_{i} \in S$. If $y_{i} \notin S$ (resp. $z_{i} \notin S$ ), then $x_{i} \in S$ and $z_{i} \in S$ (resp. $\left.y_{i} \in S\right)$ to fulfill Proposition 2.6. Clearly, exactly one of $T_{i}$ and $F_{i}$ is in $S$. If $T_{i} \in S$, then edges $F_{i} w_{i}$ and $y_{i} w_{i}$ (resp. $z_{i} w_{i}$ ) are not distinguished by $S$, a contradiction. Similar, if $F_{i} \in S$, then edges $T_{i} w_{i}$ and $y_{i} w_{i}$ (resp. $z_{i} w_{i}$ ) are not distinguished by $S$, a contradiction again. Thus, $y_{i}, z_{i} \in S$. If in addition $x_{i} \in S$, then for the triangle $w_{i} T_{i} F_{i} w_{i}$ we have a contradiction with Proposition 2.6. Therefore, $x_{i} \notin S$.

For each clause $c_{j}=y_{j}^{1} \vee y_{j}^{2} \vee y_{j}^{3}, 1 \leq j \leq m_{j}$, where $y_{j}^{k}$ is a literal in the clause $c_{j}$, we construct a satisfaction testing gadget $Y_{j}=\left(V_{j}^{\prime}, E_{j}^{\prime}\right)$, with $V_{j}^{\prime}=\left\{a_{j}^{1}, b_{j}^{1}, c_{j}^{1}, a_{j}^{2}, b_{j}^{2}, c_{j}^{2}, a_{j}^{3}, b_{j}^{3}, c_{j}^{3}\right\}$ and $E_{j}^{\prime}=\left\{a_{j}^{1} b_{j}^{1}, a_{j}^{1} c_{j}^{1}, b_{j}^{1} c_{j}^{1}, a_{j}^{2} b_{j}^{2}, a_{j}^{2} c_{j}^{2}, b_{j}^{2} c_{j}^{2}, a_{j}^{3} b_{j}^{3}, a_{j}^{3} c_{j}^{3}, b_{j}^{3} c_{j}^{3}, a_{j}^{1} a_{j}^{2}, a_{j}^{2} a_{j}^{3}, a_{j}^{3} a_{j}^{1}, b_{j}^{1} b_{j}^{2}, b_{j}^{2} b_{j}^{3}, b_{j}^{3} b_{j}^{1}, c_{j}^{1} c_{j}^{2}, c_{j}^{2} c_{j}^{3}, c_{j}^{3} c_{j}^{1}\right\}$ (see Fig. 4). Notice that the satisfaction testing gadget is isomorphic to the Cartesian product $C_{3} \square C_{3}$. 


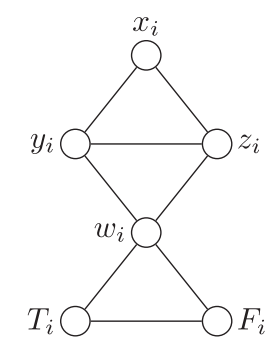

Figure 3. The truth-setting gadget for variable $u_{i}$.

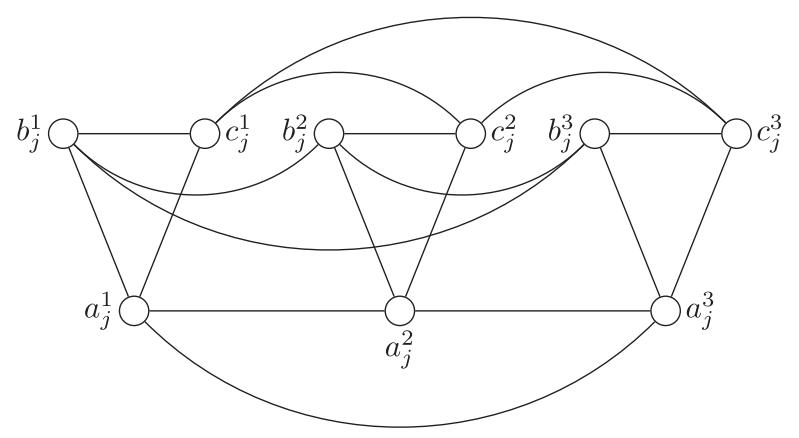

FigurE 4. The satisfaction testing gadget for clause $c_{j}$.

Claim. If $c_{j}$ is an arbitrary clause in $C$ and $Y_{j}=\left(V_{j}^{\prime}, E_{j}^{\prime}\right)$ its satisfaction testing gadget, then any incidence generator must contain at least 8 vertices from $V_{j}^{\prime}$.

Proof. Towards a contradiction, suppose there exists an incidence generator $S$ with less than 8 vertices from the satisfaction testing gadget corresponding to $c_{j}$. It follows that there exist two vertices $x, y \in V_{j}^{\prime}$ that are not in $S$. Since the diameter of $C_{3} \square C_{3}$ is 2 , the vertices $x$ and $y$ are either at a distance one or two. Every edge of $Y_{j}$ is a part of some triangle, and so $x$ and $y$ cannot be at a distance one, due to Proposition 2.6. Thus, there is a vertex $z$ such that the edges $x z$ and $z y$ exist. But those two edges are not resolved by any endpoint, a contradiction.

We also add some edges to connect the truth-setting gadgets with corresponding satisfaction testing gadgets. If a variable $u_{i}$ occurs as a literal $y_{j}^{k}$ in a clause $c_{j}=y_{j}^{1} \vee y_{j}^{2} \vee y_{j}^{3}$, then we add the following edges. If $y_{j}^{k}$ is a positive literal, then we add the edges $F_{i} b_{j}^{k}$ and $F_{i} c_{j}^{k}$. If a variable $y_{j}^{k}$ is a negative literal in a clause $c_{j}$, then we add the edges $T_{i} b_{j}^{k}$ and $T_{i} c_{j}^{k}$. For each clause $c_{j} \in C$, denote those six added edges with $E_{j}^{\prime \prime}$. We call them communication edges. Figure 5 shows the edges that were added corresponding to the clause $c_{j}=\left(\overline{u_{1}} \vee \overline{u_{2}} \vee u_{3}\right)$, where $\overline{u_{1}}$ and $\overline{u_{2}}$ represent the negative literal corresponding to the variables $u_{1}$ and $u_{2}$, respectively.

The construction of IDIM instance is then completed by setting $r=4 n+8 m$ and $G=(V, E)$, where

$$
V=\left(\bigcup_{i=1}^{n} V_{i}\right) \cup\left(\bigcup_{j=1}^{m} V_{j}^{\prime}\right)
$$

and

$$
E=\left(\bigcup_{i=1}^{n} E_{i}\right) \cup\left(\bigcup_{j=1}^{m} E_{j}^{\prime}\right) \cup\left(\bigcup_{j=1}^{m} E_{j}^{\prime \prime}\right)
$$




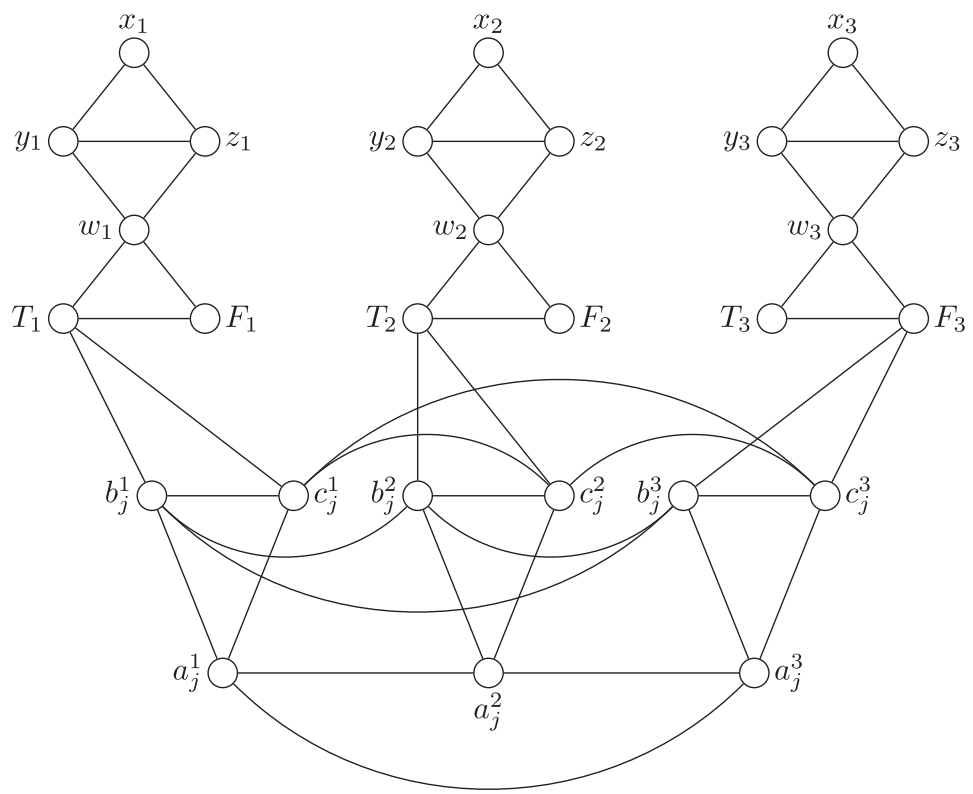

Figure 5. The subgraph associated to the clause $c_{j}=\left(\overline{u_{1}} \vee \overline{u_{2}} \vee u_{3}\right)$.

One can execute the described construction in polynomial time. Notice also that $G$ is edge-triangular.

If we show that $C$ is satisfiable if and only if $G$ has an incidence dimension less or equal than $r$, the proof of NP-completeness is completed. From Claims 3 and 3 we get the following corollary.

Corollary 3.2. The incidence dimension of $G$ constructed above is at least $r=4 n+8 m$.

The following lemmas together with Corollary 3.2 complete the proof for IDIM problem being NP-complete.

Lemma 3.3. If $C$ is satisfiable, then $\operatorname{dim}_{I}(G)=r$.

Proof. We construct an incidence generator $S$ of size $r$ based on a truth assignment of elements from the set $U$ that satisfies the collection of clauses $C$. Let $t: U \rightarrow\{$ TRUE, FALSE $\}$ be a truth assignment that satisfies the collection of clauses $C$. For each clause $c_{j}=y_{j}^{1} \vee y_{j}^{2} \vee y_{j}^{3}$, from $C$, put into $S$ the vertices $b_{j}^{k}, c_{j}^{k}$ for $k \in\{1,2,3\}$. Since the collection of clauses $C$ is satisfiable, there exists a literal $y_{j}^{k}, k \in\{1,2,3\}$, that satisfies $c_{j}$. Fix one such $k$ and put into $S$ the other two vertices $a_{j}^{\ell}$ for $\ell \in\{1,2,3\} \backslash\{k\}$.

For each Boolean variable $u_{i} \in U$, put into $S$ the vertices $\left\{y_{i}, z_{i}, w_{i}\right\}$. Also add to the set $S$, the vertex $F_{i}$ if $t\left(u_{i}\right)=$ TRUE, or the vertex $T_{i}$ if $t\left(u_{i}\right)=$ FALSE. The cardinality of the constructed set $S$ is clearly $r=4 n+8 m$.

We now take a look at the set $X=V(G) \backslash S$. For each $u_{i} \in U$ there are $x_{i}$ and exactly one of the vertices from the set $\left\{T_{i}, F_{i}\right\}$ in $X$. The distance between these two vertices is three. For each $c_{j} \in C$ exactly one of the vertices $a_{j}^{1}, a_{j}^{2}, a_{j}^{3}$ is in $X$. The vertex that is in $X$ corresponds to the variable that satisfies $c_{j}$. It follows that this vertex is at a distance three or more from all the other vertices in $X$. All other possible pairs of vertices in $X$ are also at a distance greater or equal to three. It follows that $X$ is a packing of $G$. From Corollary 2.7 follows that $S$ is an incidence generator for $G$ since $G$ is edge-triangular. Clearly, $|S|=r$ and we are done by Corollary 3.2.

Lemma 3.4. If $\operatorname{dim}_{I}(G)=r$, then the collection of clauses $C$ is satisfiable. 
Proof. Let $S$ be an arbitrary incidence generator for $G$ of cardinality $r$. The set $S$ must contain at least eight vertices from each satisfaction testing gadget and at least four vertices from each truth-setting gadget due to Claims 3 and 3. Since $|S|=r=8 m+4 n$, it follows that in $S$ there are exactly four vertices from each truthsetting component and exactly eight vertices from each satisfaction testing component. Since the graph $G$ is edge-triangular, and together with Corollary 2.7, it follows that $X=V(G) \backslash S$ is a packing for $G$. Moreover, for each $i \in\{1, \ldots, n\}$ it holds that $x_{i} \in X$ and exactly one of the vertices $T_{i}, F_{i}$ is in $X$, by Claim 3 . For each $j \in\{1, \ldots, m\}$ exactly one of the vertices $a_{j}^{1}, a_{j}^{2}, a_{j}^{3}$ is in $X$ because $b_{j}^{i}$ and $c_{j}^{i}, i \in\{1,2,3\}$, are in a common triangle with either $T_{\ell}$ or $F_{\ell}$ where $u_{\ell}$ belongs to the clause $c_{j}$.

We now define a function that satisfies all clauses from $C$. For an arbitrary $i \in\{1, \ldots, n\}$, let $v_{i} \in\left\{T_{i}, F_{i}\right\} \cap X$. Let $t: U \rightarrow\{$ TRUE, FALSE $\}$ be as follows:

$$
t\left(u_{i}\right)=\left\{\begin{array}{l}
\text { TRUE, } \quad v_{i}=T_{i} \\
\text { FALSE, } v_{i}=F_{i}
\end{array}\right.
$$

We need to show that $t$ is a satisfying truth assignment for $C$. Let $c_{j}=y_{j}^{1} \vee y_{j}^{2} \vee y_{j}^{3} \in C$ be an arbitrary clause and denote the corresponding boolean variables with $u_{j_{1}}, u_{j_{2}}, u_{j_{3}}$, respectively. To show that at least one of its literals has value TRUE, take the vertex from $V_{j}^{\prime}$ that belongs to $X$. There is exactly one of the vertices $a_{j}^{1}, a_{j}^{2}, a_{j}^{3}$ in $X$. Let $a_{j}^{k}, k \in\{1,2,3\}$, be the vertex that is in $X$. The communication edges are added in such a way that $a_{j}^{k}$ can be in $X$ (packing set) only if $u_{j_{k}}$ occurs in $c_{j}$ as:

- a positive literal and $v_{j_{k}}=T_{j_{k}}$;

- a negative literal and $v_{j_{k}}=F_{j_{k}}$.

In both cases, $c_{j}$ is satisfied by the literal corresponding to the variable $u_{j_{k}}$. It finally follows that $C$ is satisfiable, which completes the proof of this lemma.

Lemmas 3.3 and 3.4 show that the above construction is a polynomial transformation from 3-SAT to IDIM problem. Therefore, the proof of Theorem 3.1 is completed.

The proof of Theorem 3.1 yields the following result.

Corollary 3.5. The problem of finding the incidence dimension of a graph is NP-hard.

\section{Some FinAL REMARKS ON $\operatorname{dim}_{I}(G)$}

Given any graph $G$, it is easy to see that the set $V(G)$ minus one arbitrary vertex is an incident generator for $G$. On the other hand, given an incidence basis for $G$, for all but probably one edge in $E(G)$, at least one of its endpoints belongs to $S$. Moreover, for any three edges incident with the same vertex, at least two different endpoints of two different edges must be in $S$ too. In consequence, the following bounds are easy to deduce.

Remark 4.1. If $G$ is a connected graph of order $n$ with at least two edges, then

$$
\left\lfloor\frac{n}{2}\right\rfloor \leq \operatorname{dim}_{I}(G) \leq n-1 .
$$

The lower bound of Remark 4.1 is achieved for a path $P_{n}, n \in\{3,4,5,6,8\}$, a cycle $C_{4}$, a star $K_{1,3}$, and some graphs obtained by attaching a pendant vertex or an edge to some vertices of previously mentioned examples. While it is not clear if this list is complete, we can entirely describe all graphs achieving the upper bound of Remark 4.1.

Proposition 4.2. Let $G$ be a connected graph of order $n$ with at least two edges. Then $\operatorname{dim}_{I}(G)=n-1$ if and only if any two vertices of $G$ have a common neighbor. 
Proof. If there are two different vertices $x, y \in V(G)$ such that they do not have a common neighbor, then it is not difficult to see that the set $V(G)-\{x, y\}$ is an incidence generator for $G$. Thus, to have an incidence generator of order $n-1$, it is required that any two different vertices of $G$ have a common neighbor and vice versa.

Now, concerning the bounds of Remark 4.1, we next study the existence of graphs $G$ of order $n$ and incidence dimension $r$ for any $r, n$ such that $\left\lfloor\frac{n}{2}\right\rfloor \leq r \leq n-1$.

Proposition 4.3. For any integers $r, n$ with $2 \leq\left\lfloor\frac{n}{2}\right\rfloor \leq r \leq n-1$ there exists graph $G$ of order $n$ such that $\operatorname{dim}_{I}(G)=r$.

Proof. If $r=2$, then $n \in\{4,5\}$. In such a case paths $P_{4}$ and $P_{5}$, respectively, satisfy the requirements. Hence, from now on we may assume $r \geq 3$.

Consider $n$ is odd and $r=\left\lfloor\frac{n}{2}\right\rfloor<n-1$. Let $G_{r, n}$ be the graph obtained as follows.

- We begin with a complete graph $K_{r}$ with vertex set $V=\left\{u_{1}, \ldots, u_{r}\right\}$.

- Add $r+1$ vertices $w, v_{1}, \ldots, v_{r}$.

- Add the edges $u_{i} v_{i}$ for every $i \in\{1, \ldots, r\}$ and the edge $w v_{1}$.

Clearly, $G_{r, n}$ has order $2 r+1=n$. It is not difficult to see that $V$ is an incidence generator for $G_{r, n}$ and so, $\operatorname{dim}_{I}\left(G_{r, n}\right) \leq r$. Now, suppose $\operatorname{dim}_{I}\left(G_{r, n}\right)<r$ and let $S$ be an incidence basis for $G_{r, n}$. That means that there is at least one vertex $u_{j} \in V$, such that $u_{j} \notin S$. If there exists some other vertex $u_{i} \in V, i \neq j$, such that $u_{i} \notin S$, then there are two edges $u_{j} u_{k}, u_{i} u_{k}$, with $k \neq i, j$ (since $r \geq 3$ ), such that they are not incidently resolved by $S$, a contradiction. Thus, $V-\left\{u_{j}\right\} \subseteq S$, which means $|S|=r-1$ and $S=V-\left\{u_{j}\right\}$. But, in such a case, the edges $w v_{1}$ and $u_{j} v_{j}$ are not incidently resolved by $S$, which is a contradiction again. As a consequence, $\operatorname{dim}_{I}\left(G_{r, n}\right)=r$.

We next consider ( $n$ is even and $r=\left\lfloor\frac{n}{2}\right\rfloor<n-1$ ) or $\left\lfloor\frac{n}{2}\right\rfloor<r<n-1$. Let $G_{r, n}^{\prime}$ be the graph obtained as follows.

- We begin with a complete graph $K_{r}$ with vertex set $V=\left\{u_{1}, \ldots, u_{r}\right\}$.

- Add $n-r$ vertices $v_{1}, \ldots, v_{n-r}$.

- Add the edges $u_{i} v_{i}$ for every $i \in\{1, \ldots, n-r-1\}$.

- Add the edges $v_{n-r} u_{i}$ for every $i \in\{n-r, \ldots, r\}$.

- Add the edge $v_{1} v_{2}$ (notice that such two vertices always exist because $r<n-1$ ).

Clearly the order of $G_{r, n}^{\prime}$ is $n$ and we can easily notice that $V$ is an incidence generator for $G_{r, n}^{\prime}$ and so, $\operatorname{dim}_{I}\left(G_{r, n}^{\prime}\right) \leq r$. Hence, suppose $\operatorname{dim}_{I}\left(G_{r, n}^{\prime}\right)<r$ and let $S^{\prime}$ be an incidence basis for $G_{r, n}^{\prime}$. In consequence, there is at least one vertex $u_{j} \in V$ such that $u_{j} \notin S^{\prime}$. A similar procedure as earlier leads to the fact that $\operatorname{dim}_{I}\left(G_{r, n}^{\prime}\right)=r-1$ and that $S^{\prime}=V-\left\{u_{j}\right\}$. However, in this case there is an edge $u_{j} v_{l}$ for some $l \in\{1, \ldots, r\}$ such that the edges $v_{1} v_{2}$ and $u_{j} v_{l}$ are not incidently resolved by $S^{\prime}$, a contradiction. Therefore, $\operatorname{dim}_{I}\left(G_{r, n}^{\prime}\right)=r$.

We finally consider the situation $r=n-1$, which is straightforward to realize by just taking the complete graph $K_{n}$, which completes the proof.

It is natural to think that the incidence dimension is related to (edge or adjacency) dimension of graphs. Accordingly, we conclude this work by comparing $\operatorname{dim}_{I}(G)$ with $\operatorname{dim}_{\mathrm{e}}(G)$ and $\operatorname{dim}_{A}(G)$.

Proposition 4.4. For any graph $G$ without isolated vertices, $\operatorname{dim}_{I}(G) \geq \max \left\{\operatorname{dim}_{A}(G), \operatorname{dim}_{\mathrm{e}}(G)\right\}$.

Proof. Let $S$ be an incidence basis for $G$. Consider two different vertices $x, y \in V(G)-S$. If $N(x) \cap S=\emptyset$ and $N(y) \cap S=\emptyset$, then since $G$ has no isolated vertices, there are at least two edges $x x^{\prime}$ and $y y^{\prime}$ such that $x^{\prime}, y^{\prime} \notin S$. Thus, $x x^{\prime}$ and $y y^{\prime}$ are not incidently resolved by any vertex of $S$, which is a contradiction. So $N(x) \cap S \neq \emptyset$ or $N(y) \cap S \neq \emptyset$. Now, suppose $N(x) \cap S=N(y) \cap S$. Hence, there exists a vertex $w \in S$ such that the edges $x w$ 
and $y w$ are not incidently resolved by any vertex of $S$, a contradiction again. Thus, $N(x) \cap S \neq N(y) \cap S$ and, as a consequence, $S$ is an adjacency generator for $G$ and $\operatorname{dim}_{I}(G) \geq \operatorname{dim}_{A}(G)$.

Now, since any two edges $e_{1}, e_{2}$ are incident to at least two different vertices $x, y$, and at least one of $x, y$ must be in $S$, it is clear that the edges $e_{1}, e_{2}$ are distinguished by $x$ or $y$. So, $S$ is also an edge metric generator for $G$, and $\operatorname{dim}_{I}(G) \geq \operatorname{dim}_{e}(G)$, which completes the proof.

From [15] we know that $\operatorname{dim}_{A}\left(K_{r, t}\right)=r+t-2$. Also, from [18], we have $\operatorname{dim}_{e}\left(K_{r, t}\right)=r+t-2$. Now, from Proposition 2.8 (iv), we observe that the bound of Proposition 4.4 is tight. In such a case, we have equality $\operatorname{dim}_{I}\left(K_{r, t}\right)=\operatorname{dim}_{A}\left(K_{r, t}\right)=\operatorname{dim}_{e}\left(K_{r, t}\right)$. An interesting problem is then to characterize the families of graphs for which the bound of Proposition 4.4 is achieved, and moreover, finding whether the situations $\operatorname{dim}_{I}\left(K_{r, t}\right)=$ $\operatorname{dim}_{A}\left(K_{r, t}\right) \neq \operatorname{dim}_{e}\left(K_{r, t}\right), \operatorname{dim}_{I}\left(K_{r, t}\right)=\operatorname{dim}_{e}\left(K_{r, t}\right) \neq \operatorname{dim}_{A}\left(K_{r, t}\right)$ or $\operatorname{dim}_{I}\left(K_{r, t}\right)=\operatorname{dim}_{A}\left(K_{r, t}\right)=\operatorname{dim}_{e}\left(K_{r, t}\right)$ happen.

Acknowledgements. Dragana Božović, Aleksander Kelenc and Iztok Peterin are partially supported by the Slovenian Research Agency by the project No. J1-1693 and J1-9109. Ismael G. Yero has been partially supported by the Spanish Ministry of Science and Innovation through the grant PID2019-105824GB-I00.

\section{REFERENCES}

[1] N. Biggs, Perfect codes in graphs. J. Combin. Theory Ser. B 15 (1973) 289-296.

[2] D. Božović and I. Peterin, Graphs with unique maximum packing of closed neighborhoods. Discuss. Math. Graph Theory, in press (2021). DOI: 10.7151/dmgt.2304.

[3] M.P. Dobson, E. Hinrichsen and V. Leoni, Generalized limited packings of some graphs with a limited number of $P_{4}$ partners. Theor. Comput. Sci. 579 (2015) 1-8.

[4] R.D. Dutton, Global domination and packing numbers. Ars Combin. 101 (2011) 489-501.

[5] A. Estrada-Moreno, Y. Ramírez-Cruz and J.A. Rodríguez-Velázquez, On the adjacency dimension of graphs. Appl. Anal. Discrete Math. 10 (2016) 102-127.

[6] H. Fernau and J.A. Rodríguez-Velázquez, On the (adjacency) metric dimension of corona and strong product graphs and their local variants: combinatorial and computational results. Discrete Appl. Math. 236 (2018) 183-202.

[7] A. Gagarin and V. Zverovich, The probabilistic approach to limited packings in graphs. Discrete Appl. Math. 184 (2015) $146-153$.

[8] R. Gallant, G. Gunther, B. Hartnell and D.F. Rall, Limited packings in graphs. Discrete Appl. Math. 158 (2010) $1357-1364$.

[9] M.R. Garey and D.S. Johnson, Computers and Intractability: A Guide to the Theory of NP-Completeness. W. H. Freeman \& Co., New York, USA (1979).

[10] J. Geneson, Metric dimension and pattern avoidance in graphs. Discrete Appl. Math. 284 (2020) 1-7.

[11] F. Harary and R. A. Melter, On the metric dimension of a graph. Ars Combin. 2 (1976) 191-195.

[12] T.W. Haynes, S.T. Hedetniemi and P.J. Slater, Domination in Graphs. Marcel Dekker, Inc., New York, NY (1998).

[13] T.W. Haynes, S.T. Hedetniemi and P.J. Slater, Domination in Graphs: Advanced Topics. Marcel Dekker, Inc., New York, NY (1998).

[14] M.A. Henning, C. Löwenstein and D. Rautenbach, Dominating sets, packings, and the maximum degree. Discrete Math. 311 (2011) 2031-2036.

[15] M. Jannesari and B. Omoomi, The metric dimension of the lexicographic product of graphs. Discrete Math. 312 (2012) 3349-3356.

[16] K. Junosza-Szaniawski and P. Rzążewski, On the number of 2-packings in a connected graph. Discrete Math. 312 (2012) 3444-3450.

[17] A. Kelenc, D. Kuziak, A. Taranenko and I.G. Yero, On the mixed metric dimension of graphs. Appl. Math. Comput. 314 (2017) 429-438.

[18] A. Kelenc, N. Tratnik and I.G. Yero, Uniquely identifying the edges of a graph: the edge metric dimension. Discrete Appl. Math. 251 (2018) 204-220.

[19] S. Klavžar, I. Peterin and I.G. Yero, Graphs that are simultaneously efficient open domination and efficient closed domination graphs. Discrete Appl. Math. 217 (2017) 613-621.

[20] M. Knor, S. Majstorović, A.T. Masa Toshi, R. Škrekovski and I.G. Yero, Graphs with the edge metric dimension smaller than the metric dimension. Appl. Math. Comput. 401 (2021) 126076.

[21] M. Knor, R. Škrekovski and I.G. Yero, A note on the metric and edge metric dimensions of 2-connected graphs. Discrete Appl. Math., in press. DOI: 10.1016/j.dam.2021.02.020.

[22] J. Kratica, V. Filipovic and A. Kartelj, Edge metric dimension of some generalized Petersen graphs. Results Math. 74 (2019) 182. 
[23] D. Kuziak and I.G. Yero, Metric dimension related parameters in graphs: A survey on combinatorial, computational and applied results. Preprint arXiv:2107.04877 (2021).

[24] A. Meir and J.W. Moon, Relations between packing and covering numbers of a tree. Pacific J. Math. 61 (1975) $225-233$.

[25] D.A. Mojdeh, B. Samadi, A. Khodkar and H.R. Golmohammadi, On the packing numbers in graphs. Australas. J. Combin. 71 (2018) 468-475.

[26] I. Peterin and I.G. Yero, Edge metric dimension of some graph operations. Bull. Malays. Math. Sci. Soc. 43 (2020) $2465-2477$.

[27] I. Sahul Hamid and S. Saravanakumar, Packing parameters in graphs. Discuss. Math. Graph Theory 35 (2015) 5-6.

[28] P.J. Slater, Leaves of trees. Congressus Numerantium 14 (1975) 549-559.

[29] R.C. Tillquist, R.M. Frongillo and M.E. Lladser, Getting the lay of the land in discrete space: A survey of metric dimension and its applications. Preprint arXiv:2104.07201 (2021)

[30] N. Zubrilina, On the edge dimension of a graph. Discrete Math. 341 (2018) 2083-2088.

\section{Subscribe to Open (S2O) A fair and sustainable open access model}

This journal is currently published in open access under a Subscribe-to-Open model (S2O). S2O is a transformative model that aims to move subscription journals to open access. Open access is the free, immediate, online availability of research articles combined with the rights to use these articles fully in the digital environment. We are thankful to our subscribers and sponsors for making it possible to publish this journal in open access, free of charge for authors.

\section{Please help to maintain this journal in open access!}

Check that your library subscribes to the journal, or make a personal donation to the S2O programme, by contacting subscribers@edpsciences.org

More information, including a list of sponsors and a financial transparency report, available at: https://www. edpsciences.org/en/maths-s2o-programme 